\title{
Fast Direct Solution of the Combined Field Integral Equation
}

\author{
Alex Heldring ${ }^{\# 1}$, José Maria Tamayo ${ }^{\# 2}$ and Juan Manuel Rius ${ }^{\# 3}$

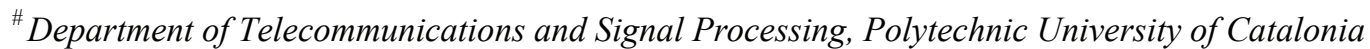 \\ C. Jordi Girona, 31, 08034, Barcelona, Spain \\ ${ }^{1}$ heldring@tsc.upc.edu \\ jose.maria.tamayo@tsc.upc.edu \\ rrius@tsc.upc.edu
}

\begin{abstract}
The Method of Moments for electromagnetic scattering and radiation problems is often used in conjunction with the EFIE because the EFIE allows the analysis of open surfaces. For electrically large closed surfaces, the CFIE is often much more efficient because it yields a well conditioned impedance matrix. This is particularly important when an iterative solution method is used. This paper compares the EFIE and the CFIE for a novel fast direct (non-iterative) solution method, the Multiscale Compressed Block Decomposition method.
\end{abstract}

\section{INTRODUCTION}

The Method of Moments [1] is a popular tool for the simulation of electromagnetic radiation and scattering. Often it is applied to a surface integral equation (SIE) which is discretized using local basis functions defined on a triangulation of the structure under investigation. If this structure consists exclusively of perfectly conducting surfaces then the typical SIE that are used are the Electric Field Integral Equation (EFIE), the Magnetic Field Integral Equation (MFIE) or a combination of the two (CFIE).

The MoM leads to a dense linear system, with a direct solution time that scales with the cube of the number of basis functions $N$, or, for a discretization that is fixed with respect to the wave length, with the sixth power of the frequency. This proportionality is referred to as the computational complexity of the method. Evidently this poses a strict limit to the maximum electrical size of the problem under investigation.

Many methods to accelerate the solution have been proposed over the past decades, most of them using an approximate representation of the MoM impedance matrix which can be used in an iterative solution algorithm. The most famous among these methods is the Multilevel Fast Multipole Method (MLFMM) [2] which reduces the complexity to $K N \log N$ where $K$ is the required number of iteration steps. Although these methods have brought an enormous gain in efficiency for electrically large problems, they typically have a number of drawbacks. To start with, many problems lead to a badly conditioned impedance matrix. Iterative methods then need to be preconditioned to converge in a reasonable number of steps. But the choice, parameter adjustment and construction of a good preconditioner can be a tedious and unpredictable process. Another drawback is that for every single independent vector, the iteration must be restarted. Furthermore, some of the most efficient iterative methods, such as MLFMM, are kernel dependent. In short, a direct (non-iterative) solution method with a reduced complexity remains an interesting alternative, even if it is asymptotically less efficient than the above mentioned iterative methods.

In [3] we have presented such a method, the Compressed Block Decomposition (CBD). In [4] we showed that the complexity of this method is $N^{2} \log ^{2} N$. In [5] we presented a multiscale version of the CBD applied to static or lowfrequency problems which was shown to yield an $\log ^{3} N$ complexity. Due to the oscillatory kernel this is not true in the high frequency regime, and in fact the multiscale method from [5] needs several adaptations to be efficient at high frequencies. In [6] we present a multiscale extension to the $\mathrm{CBD}$ for the high frequency regime (MS-CBD) and demonstrate with a numerical example that it is superior to the single-scale CBD. Its primary feature is that, just like singlelevel $\mathrm{CBD}$, it is entirely algebraic, since it exclusively uses ACA [7], truncated SVD and QR decompositions to achieve block-wise compression of the impedance matrix and maintain it after factorization. In the paper we also show that the MS-CBD has $N^{2}$ complexity and is therefore asymptotically (with respect to frequency) superior to the CBD.

Although by avoiding an iterative algorithm we much alleviate the problems presented by badly conditioned matrices, the MS-CBD is not insensitive to the impedance matrix condition number. A consequence of a high condition number is that small perturbations in any of the matrix elements may cause large errors in the solution. Since the block-compression through ACA and truncated SVD involves an approximation (governed by a threshold $\tau$ on the singular values), a badly conditioned matrix requires a lower threshold and therefore less compression.

The EFIE is the most commonly used formulation for perfect conductors, since it is applicable to both closed and open geometries (open geometries have edges). The MFIE and therefore the CFIE are restricted to closed geometries. However, the EFIE, besides suffering from a well known lowfrequency breakdown [8], is also often badly conditioned. This is in contrast with the MFIE and with the CFIE to an even greater extent, which are well conditioned independently of the electrical size of the problem. 
We expect that, although the compression rate will not be better for CFIE than for EFIE as a function of the threshold value, CFIE will allow higher threshold values for a given accuracy. It may therefore be expedient to use CFIE when the problem geometry allows it (closed problems), even taking into account that the MS-CBD fully profits from the symmetry of the EFIE impedance matrix, yielding a gain of almost a factor two in computation time.

\section{Multi ScAle-Compressed Block Decomposition.}

The MS-CBD is essentially a nested implementation of the well known Partitioned Matrix Inverse formulas [9]. For a matrix $\mathrm{Z}$ partitioned into four blocks:

$$
Z^{-1}=\left[\begin{array}{ll}
\tilde{Z}_{11} & \tilde{Z}_{12} \\
\tilde{Z}_{21} & \tilde{Z}_{11}
\end{array}\right],
$$

with

$$
\begin{gathered}
\widetilde{Z}_{11}=Z_{11}^{-1}+Z_{11}^{-1} Z_{12} \widetilde{Z}_{22} Z_{21} Z_{11}^{-1} \\
\widetilde{Z}_{12}=-Z_{11}^{-1} Z_{12} \widetilde{Z}_{22} \\
\widetilde{Z}_{21}=-\widetilde{Z}_{22} Z_{21} Z_{11}^{-1} \\
\widetilde{Z}_{22}=\left(Z_{22}-Z_{21} Z_{11}^{-1} Z_{12}\right)^{-1}
\end{gathered}
$$

The first step is the construction of the impedance matrix in compressed form. The basis functions are subdivided recursively into groups based on geometrical proximity. All matrix blocks, at any scale, that are not on the diagonal, are compressed into truncated SVD form. This means that the SVD is computed and all columns and rows in the three SVD factors that relate to singular values smaller than $\tau$ times the largest singular value are discarded. The computation of the SVD, which is an expensive operation, is accelerated by using the ACA algorithm. Blocks on the diagonal are either subdivided again, or, but only at the smallest scale, inverted directly by LU decomposition.

The second step is the inversion of the compressed matrix according to (1). As equation (2) shows, the only operations involved are multiplication and summation of matrices (and inversion, but this is recursively done by the same MS-CBD algorithm, except at the smallest scale). To maintain the compression, every multiplication and summation requires a restoration of the SVD form of the result. This is achieved with a number of QR and truncated SVD decompositions. The details of the algorithm will be published in [6].

The last step is a back substitution (similar to LU back substitution) to obtain the solution for one or several independent (excitation) vectors. This last step is typically orders of magnitude cheaper than the previous ones, even for thousands of excitation vectors.

\section{NUMERICAL RESULTS.}

In order to investigate the respective performance of the MS-CBD with the EFIE and the CFIE formulations, we computed the monostatic RCS of a perfectly conducting cube for a range of threshold values in both formulations. We chose to compute the monostatic RCS since this represents the typical usage of the MS-CBD (one of the major advantages of MS-CBD in comparison with iterative methods is the very small overhead for multiple excitation vectors). The setup of the experiment is shown in Fig. 1. We did the computations at two frequency scales, a small cube (rib-size 1 $\lambda$ ) and a larger one (rib-size $8 \lambda$ ) under otherwise identical conditions in order to see whether the result is dependent on the overall electrical size of the problem. In both cases, RWG basis functions with a mean edge size of $0.07 \lambda$ were used. For the small problem, this meant $N=4,608$ basis functions, the large problem needed $N=294,912$ basis functions.

The small problem was done with four-level MS-SBD, the large problem with ten-level MS-CBD. The incident field is polarized along the $\mathrm{z}$-axis and propagates in the $\mathrm{XY}$ plane at angles between $\phi=0^{\circ}$ and $\phi=45^{\circ}$ (50 incidence angles for the small problem, 1,000 incidence angles for the large problem).

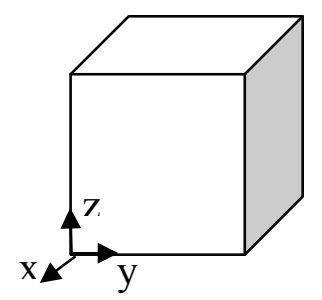

Fig. 1. PEC cube in free space.

In Figs 2 and 3 the monostatic RCS is shown for the small problem, for EFIE and CFIE respectively. Fig. 4 and 5 show the same for the large problem. The overall conclusion is that the EFIE result is accurate for a threshold value $\tau=10^{-3}$, while the CFIE result is already accurate with $\tau=10^{-2}$. This is true in both cases indicating that the size of the problem does not play a significant role.

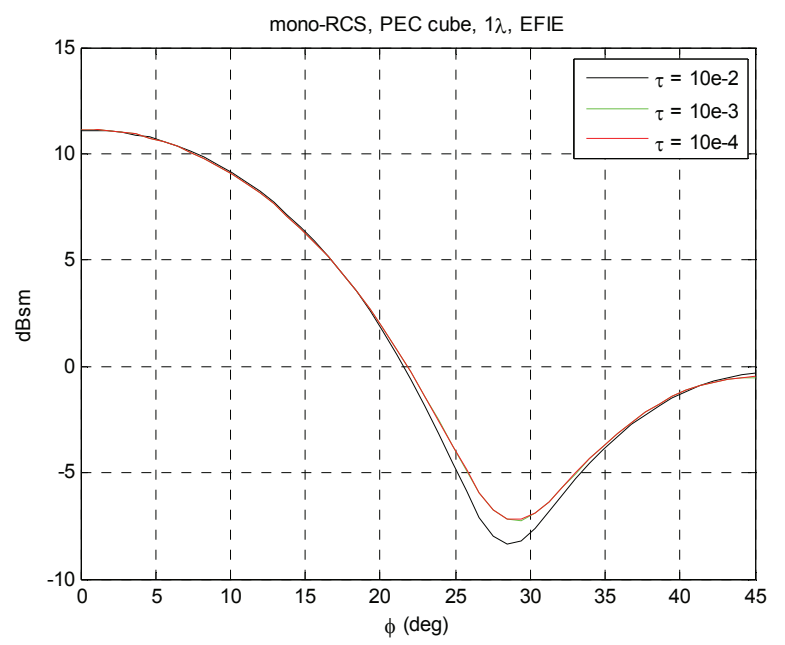

Fig. 2. EFIE MS-CBD computation of monostatic RCS, PEC cube, 4,608 basis functions, 50 incidence angles. 


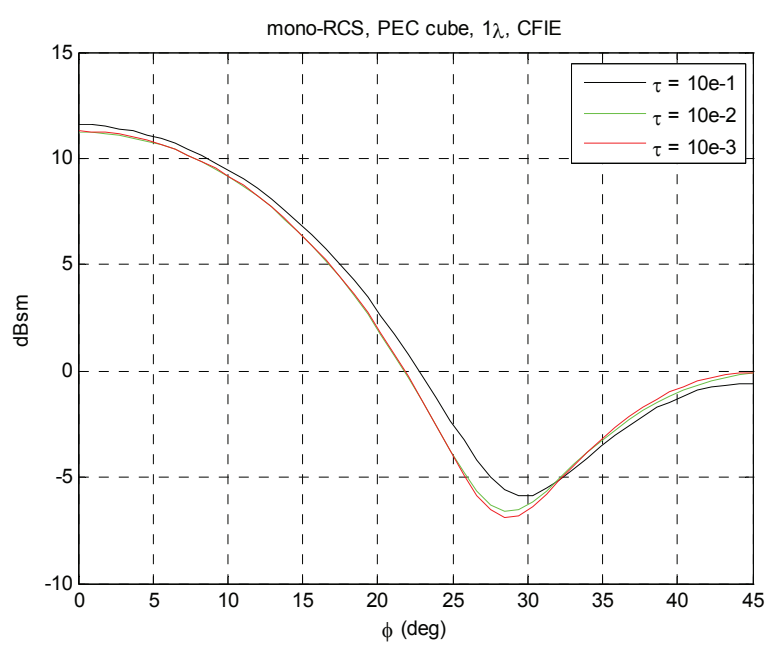

Fig. 3. CFIE MS-CBD computation of monostatic RCS, PEC cube, 4,608 basis functions, 50 incidence angles.

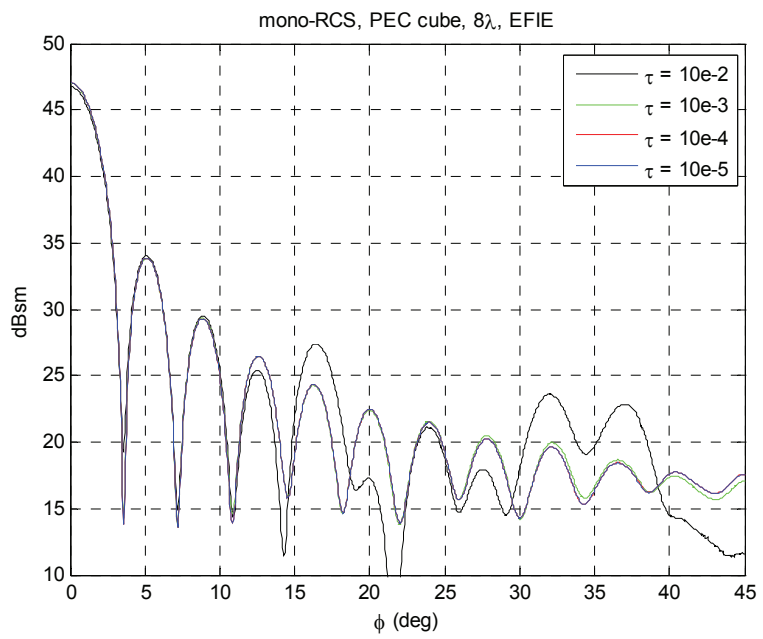

Fig. 4. EFIE MS-CBD computation of monostatic RCS, PEC cube, 294,912 basis functions, 1,000 incidence angles.

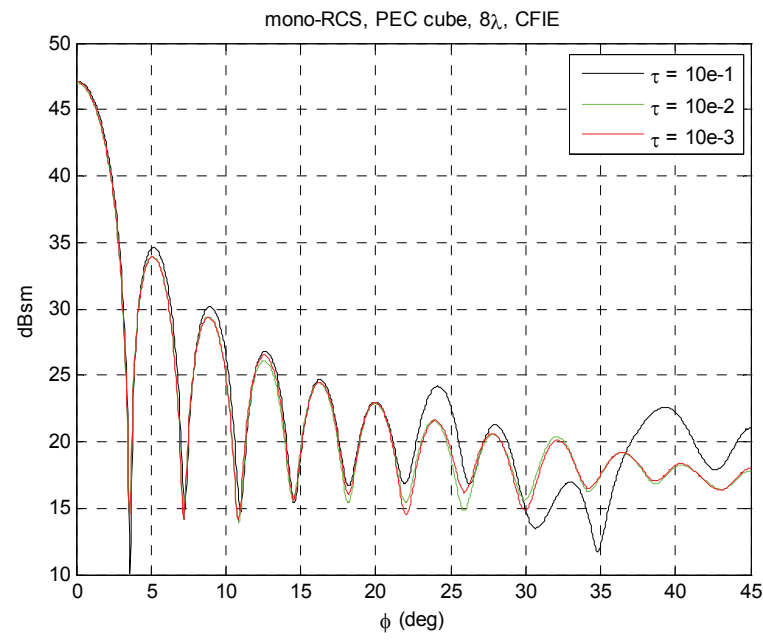

Fig. 5. CFIE MS-CBD computation of monostatic RCS, PEC cube, 294,912 basis functions, 1,000 incidence angles.
Tables I to IV show the computation times and storage requirements for the different computations. All the computations were performed on a PC with a Quad Intel(R) Xeon(R) X5482 processor at $3.20 \mathrm{GHz}$ and 64 GB of memory. The code was implemented in Matlab, in single precision.

The EFIE formulation fully exploited the symmetry of the EFIE impedance matrix, resulting in a gain of a factor two in the matrix build time, inverse build time, matrix size and inverse size (not in the back substitution time). The CFIE does not have this property.

TABLE I

EFIE, $N=4,608$ AND 50 INCIDENCE ANGLES

\begin{tabular}{|l|l|l|l|}
\hline & $\tau=10^{-2}$ & $\tau=10^{-3}$ & $\tau=10^{-4}$ \\
\hline$Z$ build (sec) & 6.8 & 11.6 & 15.9 \\
\hline$Z^{-1}$ build (sec) & 1.6 & 2.3 & 3.6 \\
\hline Backsub (sec) & 0.3 & 0.3 & 0.4 \\
\hline$Z$ Size (MB) & 25 & 33 & 38 \\
\hline$Z^{-1}$ Size (MB) & 20 & 27 & 34 \\
\hline
\end{tabular}

TABLE III

CFIE, $N=4,608$ AND 50 INCIDENCE ANGLES

\begin{tabular}{|l|l|l|l|}
\hline & $\tau=10^{-1}$ & $\tau=10^{-2}$ & $\tau=10^{-3}$ \\
\hline$Z$ build (sec) & 7.7 & 14.6 & 24.5 \\
\hline$Z^{-1}$ build (sec) & 1.8 & 2.7 & 4.2 \\
\hline Backsub (sec) & 0.3 & 0.3 & 0.3 \\
\hline$Z$ Size (MB) & 23 & 40 & 55 \\
\hline$Z^{-1}$ Size (MB) & 25 & 41 & 63 \\
\hline
\end{tabular}

TABLE IIIII

EFIE, $N=294,912$ AND 1,000 INCIDENCE ANGLES

\begin{tabular}{|l|l|l|l|l|}
\hline & $\tau=10^{-2}$ & $\tau=10^{-3}$ & $\tau=10^{-4}$ & $\tau=10^{-5}$ \\
\hline$Z$ build (sec) & 1,816 & 3,059 & 4,297 & 5,991 \\
\hline$Z^{-1}$ build (sec) & 1,249 & 2,140 & 3,379 & 6,283 \\
\hline Backsub (sec) & 57 & 76 & 100 & 167 \\
\hline$Z$ Size (MB) & 4,937 & 6,416 & 7,838 & 9,479 \\
\hline$Z^{-1}$ Size (MB) & 4,909 & 7,186 & 9,743 & 15,401 \\
\hline
\end{tabular}

TABLE IVV

CFIE, $N=294,912$ AND 1,000 INCIDENCE ANGLES

\begin{tabular}{|l|l|l|l|}
\hline & $\tau=10^{-1}$ & $\tau=10^{-2}$ & $\tau=10^{-3}$ \\
\hline$Z$ build (sec) & 2,197 & 3,890 & 6,266 \\
\hline$Z^{-1}$ build (sec) & 929 & 1,942 & 3,601 \\
\hline Backsub (sec) & 35 & 47 & 61 \\
\hline$Z$ Size (MB) & 5,736 & 8,910 & 11,621 \\
\hline$Z^{-1}$ Size (MB) & 5,611 & 9,551 & 14,184 \\
\hline
\end{tabular}

The Tables show that both the computation times and the matrix sizes grow very significantly for lower threshold values. In terms of efficiency it is therefore quite expedient to know the maximum threshold for a given desired accuracy. It is however difficult to establish a general rule for this.

Another conclusion from the Tables is that, accounting for the factor two gain in the EFIE due to symmetry, the matrix sizes of EFIE and CFIE are approximately equal for equal threshold value. This is to be expected since the principal limitation to the compression rate is the oscillatory Greens function, which is present in both cases.

Finally, with respect to the suggestion in the introduction that CFIE may be an interesting option for MS-CBD due to 
the superior conditioning; these results indicate that in fact, the advantage does not outweigh the gain from the symmetry of the EFIE, as a comparison of the performance for converged results in both cases shows.

\section{CONCLUSION}

A comparison of the performance of the Multiscale Compressed Block Decomposition (MS-CBD) in combination with the EFIE and with the CFIE has been carried out. The results indicate that the advantage due to the better conditioning of the impedance matrix for the CFIE is important but does not outweigh the gain of a factor two due to the symmetry of the EFIE impedance matrix. This conclusion is valid for the MS-CBD since it is a direct (noniterative) method and therefore not critically dependent on the matrix condition number. If the same block-compressed impedance matrix is used within an iterative method, then the CFIE will often be much more efficient because the convergence of any iterative method is highly dependent on the matrix condition number.

\section{ACKNOWLEDGMENT}

This work was supported by the Spanish Interministerial Commission on Science and Technology (CICYT) under projects TEC2009-13897-C03-01 and TEC2007-66698-C0401/TCM and CONSOLIDER CSD2008-00068 and by the "Ministerio de Educación y Ciencia" through the FPU fellowship program.

\section{REFERENCES}

[1] R. F. Harrington, Field Computation by Moment Methods, MacMillan New York, 1968.

[2] J. Song, C.C. Lu and W.C. Chew, .Multilevel Fast Multipole Algorithm for Electromagnetic Scattering by Large Complex Objects., IEEE Trans.on Antennas and Propag., Vol. 45, No. 10, pp. 1488-1493, October 1997

[3] A. Heldring, J. M. Rius,J. M. Tamayo, J. Parrón, E. Ubeda, .Fast Direct Soluton of Method of Moments Linear System,. IEEE Trans. on Antennas and Propag., Vol 55, No 11, pp. 3220-3228, Nov 2007

[4] A. Heldring, J. M. Rius, J. M. Tamayo, 'Comments on Fast Direct Soluton of Method of Moments Linear System,' accepted for publication in IEEE Trans. on Antennas and Propag.

[5] A. Heldring, J.M. Rius, J.M. Tamayo and J. Parron, 'Compressed Block Decomposition Algorithm for Fast Capacitance Extraction,'. IEEE Trans. on Computer Aided Design, Vol. 27, No. 2, pp 265-271, Feb 2008

[6] A. Heldring, J. M. Rius, J. M. Tamayo, 'Multiscale Compressed Block Decomposition for Fast Direct Solution of Method of Moments Linear System,' submitted for publication in IEEE Trans. on Antennas and Propag.

[7] M. Bebendorf, .Approximation of boundary element matrices,., Numer. Math. (2000) 86: 565.589

[8] Y. Chu, W. C. Chew, J. Zhao and S. Chen, 'Surface Integral Equation Formulation for Low-Frequency Scattering From a Composite Object,' IEEE Trans. on Antennas and Propag., Vol. 51, No. 10, pp 2837-2844, Oct 2003

[9] T. Banachiewicz, .Zur Berechnung der Determinanten, wie auch der Inversen und zur darauf basierten Aufosung der Systeme linearer Gleichungen,. Acta Astronom. Ser. C 3, pp. 41.67, 1937 